\title{
CULTURA DA AVALIAÇÃO E CONTEXTOS DIGITAIS DE APRENDIZAGEM: 0 MODELO PrACT
}

\author{
CULTURE OF EVALUATION AND DIGITAL CONTEXTS OF LEARNING: THE \\ MODEL PrACT
}

\section{CULTURA DE LA EVALUACIÓN Y CONTEXTOS DIGITALES DE APRENDIZAJE: EL MODELO PRACT}

Lúcia Amante ${ }^{1}$ Isolina Oliveira ${ }^{2}$

Alda Pereira ${ }^{3}$

Resumo: Com os novos cenários de aprendizagem decorrentes do desenvolvimento tecnológico e em particular da Web 2.0, torna-se necessário repensar o processo de ensinoaprendizagem e as práticas docentes, designadamente ao nível de estratégias de avaliação alinhadas com paradigmas mais recentes, onde novos conceitos e perspetivas dão lugar a uma nova cultura de avaliação. Esta nova perspetiva, opõe-se à "cultura psicométrica do teste" e tem por base o construtivismo social, encarando o estudante como um participante ativo que partilha a responsabilidade pelo processo de aprendizagem, que se envolve na avaliação que, por sua vez, deve assumir múltiplas e diversificadas formas. Que novos conceitos emergem nesta abordagem? Que modelos podem ancorar esta nova cultura de avaliação? Como desenhar um sistema de avaliação que garanta a qualidade desta nova abordagem? Como podem os novos media digitais contribuir para uma avaliação mais relevante? Partindo do trabalho empírico desenvolvido em pesquisas neste âmbito e do quadro conceptual do modelo PrACT (Praticabilidade, Consistência, Autenticidade e Transparência) (PEREIRA, OLIVEIRA \& TINOCA, 2011; PEREIRA, OLIVEIRA \& AMANTE, 2015) assume-se a cultura da avaliação como contraponto à cultura do teste e propõe-se uma abordagem edumétrica na avaliação da qualidade das novas formas de avaliação. Neste texto argumentamos sobre a importância de desenvolver e aplicar um quadro conceptual, com base no conceito de avaliação digital alternativa, que enquadre as práticas de avaliação e contribua para a sustentabilidade dessas práticas designadamente nos novos contextos de aprendizagem.

Palavras chave: aprendizagem; avaliação digital alternativa; cultura da avaliação; edumetria.

Submetido em: 23/10/2017 - Aceito em: 04/11/2017 - Publicado em: 13/11/2017.

${ }^{1}$ Universidade Aberta, LE@D, Lucia.Amante@uab.pt

${ }^{2}$ Universidade Aberta, LE@D, Isolina.Oliveira@uab.pt

${ }^{3}$ Universidade Aberta, LE@DAlda.Pereira@uab.pt 


\section{Revista Docência e Cibercultura}

Abstract: With the new learning scenarios arising from technological development and in particular of Web 2.0, it becomes necessary to rethink the teaching-learning process and teaching practices, namely at the level of evaluation strategies aligned with more recent paradigms, where new concepts and perspectives give way to a new evaluation culture. This new perspective opposes the "psychometric culture of the test" and is based on social constructivism, viewing the student as an active participant who shares responsibility for the learning process, which is involved in the evaluation that, in turn, must take on multiple and diversified forms. What new concepts emerge in this approach? What models can anchor this new evaluation culture? How to design an evaluation system that guarantees the quality of this new approach? How can new digital media contribute to a more relevant assessment? Based on the empirical work developed in researches in this field and on the conceptual framework of the PrACT (Practicability, Consistency, Authenticity and Transparency) model (PEREIRA, OLIVEIRA \& TINOCA, 2011; PEREIRA, OLIVEIRA \& AMANTE, 2015) counterpoint to the culture of the test and an edumetric approach is proposed in the evaluation of the quality of the new forms of evaluation. In this text, we argue about the importance of developing and applying a conceptual framework, based on the concept of alternative digital evaluation, that frames the evaluation practices and contributes to the sustainability of these practices, especially in the new learning contexts.

Keywords: learning; alternative digital evaluation; evaluation culture; edumetry.

Resumen: Con los nuevos escenarios de aprendizaje derivados del desarrollo tecnológico y en particular de la Web 2.0, es necesario repensar el proceso de enseñanza-aprendizaje y las prácticas docentes, en particular a nivel de estrategias de evaluación alineadas con paradigmas más recientes, donde nuevos conceptos y perspectivas dan lugar a una nueva cultura de evaluación. Esta nueva perspectiva, se opone a la "cultura psicométrica de la prueba" y tiene como base el constructivismo social, encarando al estudiante como un participante activo que comparte la responsabilidad por el proceso de aprendizaje, que se involucra en la evaluación que, a su vez, debe asumir múltiples y diversificadas formas. ¿Qué nuevos conceptos emergen en este enfoque? ¿Qué modelos pueden anclar esta nueva cultura de evaluación? ¿Cómo diseñar un sistema de evaluación que garantice la calidad de este nuevo enfoque? ¿Cómo pueden los nuevos medios digitales contribuir a una evaluación más relevante? En el trabajo empírico desarrollado en investigaciones en este ámbito y del marco conceptual del modelo PrACT (Practicabilidad, Consistencia, Autenticidad y Transparencia) (PEREIRA, OLIVEIRA \& TINOCA, 2011, PEREIRA, OLIVEIRA \& AMANTE, 2015) se asume la cultura de la evaluación como contrapunto a la cultura de la prueba y se propone un abordaje edumétrico en la evaluación de la calidad de las nuevas formas de evaluación. En este texto se argumenta sobre la importancia de desarrollar y aplicar un marco conceptual basado en el concepto de evaluación digital alternativa que enmarca las prácticas de evaluación y contribuye a la sostenibilidad de estas prácticas, en particular en los nuevos contextos de aprendizaje.

Palabras clave: aprendizaje; evaluación digital alternativa; cultura de la evaluación; edumetría. 
INTRODUÇÃO

Os novos contextos digitais têm vindo a influenciar todas as esferas sociais e também a esfera educativa, apesar de os contextos educativos serem tradicionalmente resistentes à mudança. A Web 2.0 em particular, mudou radicalmente a forma como as pessoas utilizam a internet e interagem com os outros, mas também a forma como lidam com a informação e com o conhecimento refletindo-se esses comportamentos nos cenários educativos. De meros consumidores de conteúdos e de informação, os utilizadores passaram também a ser produtores, criando conteúdos que partilham através do software social. Estes passam a fazer parte do corpus de informação e de conhecimento disponíveis na Web, dando lugar também a um novo conjunto de espaços, processos e cenários de aprendizagem.

Importa assim pensar a avaliação pedagógica nestes novos cenários, porque avaliar é fundamental por proporcionar dados sobre o desempenho dos estudantes com vista ao reconhecimento de aprendizagens ou validação de competências, mas também pelas informações que proporciona a todos os atores envolvidos neste processo, designadamente professores e alunos. Estas informações permitem ajustar práticas pedagógicas, designadamente tomar decisões sobre o tipo de feedback a dar ao estudante, acentuando o caráter formador da avaliação e a regulação dos processos de ensino e aprendizagem. Também as instituições carecem de modelos de avaliação pedagógica que assegurem a qualidade dessas práticas e que se constituam como uma referência.

Como é sabido, tradicionalmente a avaliação centrou-se primordialmente na sua função de classificação e certificação. Contudo, em especial nas duas últimas décadas, o balanço entre as diversas funções da avaliação concedeu maior destaque à sua função formativa (BOUD, 1995; HADJI, 1997, FERNANDES, 2004; PINTO \& SANTOS, 2006).

As tecnologias digitais e o seu crescente uso vieram acelerar a transição para outras formas de avaliação, permitindo trabalhar com uma diversidade de ferramentas e atividades que centram a aprendizagem no estudante e proporcionam múltiplas formas de interação, designadamente a colaborativa. Deste modo, as limitações dos modelos tradicionais de avaliação foram ultrapassadas por um novo paradigma para a avaliação, nomeadamente em 
ambientes de aprendizagem online. Com efeito, o uso das tecnologias faculta uma maior adaptabilidade e flexibilidade do que a oferecida na avaliação tradicional, baseada por norma em testes escritos, sobre cuja validade se foi perdendo confiança dada a sua natureza tantas vezes irrealista e a sua sobrevalorização enquanto objetivo final do processo de ensino (McDOWELL, 1995).

Na nossa atividade docente, também ligada à investigação nesta área em contexto de aprendizagem online no ensino superior, sentimos necessidade de ultrapassar práticas tradicionais de avaliação centradas nos conteúdos e na mera função de classificação. Propusemo-nos pensar um quadro conceptual da avaliação que fosse ao encontro da prática pedagógica desenvolvida nestes novos contextos de aprendizagem, enquadrada pelo modelo pedagógico virtual da Universidade Aberta, em Portugal. Este modelo pedagógico (PEREIRA et al. 2007) assenta em quatro grandes princípios, designadamente a aprendizagem centrada no estudante e o primado da interação, valorizando a avaliação contínua e incentivando práticas de avaliação alternativa, mediadas e potenciadas pelos recursos digitais disponíveis.

\section{NOVA CULTURA DA AVALIAÇÃO E PERSPETIVA EDUMÉTRICA}

A influência do paradigma construtivista no modo de encarar a avaliação vem dar lugar ao papel do contexto que emerge como facilitador ou inibidor do processo de comunicação em que a avaliação se desenvolve. "Um olhar atento para a avaliação faz emergir a dimensão comunicacional da avaliação, onde o avaliador e avaliado são actores num processo de comunicação social específico". (PINTO \& SANTOS, 2006, p. 24), configurando-se como um diálogo, uma "acção feita por pessoas e para pessoas sendo o seu principal objetivo encontrar respostas em situação dinâmica, para melhorar o desenvolvimento, tanto das ações, como das relações numa dada situação social" (Op. Cit. p. 37). O professor assume um papel de organizador dos contextos que acompanha os estudantes nas aprendizagens, sendo usadas diversas formas de trabalhar que vão desde o trabalho em grupo ao trabalho autónomo direcionado para a sistematização dos novos conhecimentos. $\mathrm{O}$ aluno assume-se também como agente da sua própria avaliação, a 


\section{Revista Docência e Cibercultura}

autoavaliação emerge como uma forma de avaliação essencial, assente na definição e negociação dos critérios de avaliação que constituem, assim, um referencial neste processo. O aluno passa a ser ele próprio agente regulador da sua aprendizagem e a avaliação ganha um novo significado. Para além desta forma de avaliação, intrinsecamente ligada à aprendizagem, outros meios de avaliação são utilizados, mantendo-se a função de classificação, seleção e certificação imposta pelos sistemas educativos.

Perspetivas mais recentes sublinham um novo propósito da avaliação, ligado à necessidade de avaliar competências. BOUD (2000) propõe a designação de "avaliação sustentável", questionando em que medida a avaliação tem um papel na preparação dos estudantes para a aprendizagem ligada à vida profissional, assinalando que muita da avaliação que se faz é inadequada para a tarefa de preparar os estudantes para o mundo real em que serão chamados a atuar. Também autores como Baartman, Bastiaens, Kirschner e van der Vleuten, (2007), Sluijsmans, Prins e Martens (2006) bem como Pereira, Oliveira e Tinoca (2011) suscitam a discussão em torno das implicações que as questões sobre a aprendizagem ao longo da vida têm no design dos programas de avaliação.

A avaliação de competências requer pois uma nova abordagem, na qual conhecimentos, capacidades e atitudes estão integrados (BAARTMAN et al., 2007), implicando, necessariamente, o recurso a uma variedade de estratégias e modos de avaliação (DIERICK \& DOCHY, 2001; McCONNELL, 2004; MacLELLAN, 2004), que permitam contemplar o desempenho em tarefas autênticas, representativas dos contextos reais, nos quais as competências deverão ser aplicadas.

Assim, um dos grandes desafios que se coloca atualmente às instituições universitárias envolve alterar radicalmente a sua forma de desenvolvimento curricular, passando de um currículo baseado em conteúdos para um currículo baseado em competências, flexível e interdisciplinar. Nesse sentido, os contextos virtuais e os novos recursos de aprendizagem têm vindo a contribuir para o repensar das estratégias de avaliação. Desde a multiplicidade de forma (testes de escolha múltipla online, quizzes, participação em fóruns electrónicos, trabalho de grupo online, blogs e e-portefólios, entre outros (cf. (TINOCA, OLIVEIRA \& PEREIRA, 2007; GOMES, 2010; AMANTE, 20011) à adoção de práticas de auto, hetero e coavaliação (BOUD, 1995; FALCHIKOV, 2005; McCONNELL, 
2006), são várias as novas possibilidades que podem contribuir para uma avaliação mais significativa e mais próxima do real.

Na sociedade de conhecimento, as exigências já não são as da sociedade industrial que determinaram a criação do modelo de Escola transmissiva que ainda hoje prevalece, em larga medida. O paradigma psicométrico, que durante décadas sustentou a prática da avaliação, assentava numa conceção de aprendizagem e de avaliação baseada no "drill and practice". Na atual sociedade há outras exigências. Num mundo imprevisível e em permanente mudança pretende-se que os cidadãos sejam capazes de desenvolver conhecimento de forma autónoma, que sejam reflexivos, criativos, críticos, que consigam resolver problemas complexos e que se corresponsabilizem pela sua própria aprendizagem (FIGUEIREDO, 2016). Neste âmbito, precisamos de um novo paradigma de ensino onde o que é determinante é a construção de ambientes ricos que promovam a aprendizagem em ligação com os contextos reais. Tendo em conta estes cenários, "a avaliação tem que ir para além da medição da reprodução de conhecimento" (DIERICK \& DOCHY, 2001, p. 301). Surge de modo imperativo, a necessidade da utilização de um novo desenho de avaliação que dá lugar a uma nova abordagem conhecida como a "cultura da avaliação", por contraponto à "cultura do teste".

Esta cultura da avaliação caracteriza-se essencialmente por:

- Dar ênfase à integração da avaliação no ensino (BIRENBAUM, 1996);

- Envolver o estudante no desenvolvimento da sua própria avaliação em diálogo com o professor (McCONNELL, 2006);

- Avaliar tanto o processo como o produto;

- Assumir diversas formas, não estandardizadas, associadas e interligadas com as práticas de ensino (DOCHY, 2001);

- Utilizar uma variedade de tarefas de avaliação próximas de situações da vida real (HERRINGTON \& HERRINGTON, 1998);

- Assumir um cunho investigativo (GULIKERS et al., 2004;)

- Reforçar a reflexão dos estudantes sobre as suas aprendizagens (PINTO, 2016; McCONNELL, 2006);

- Valorizar uma descrição qualitativa em detrimento de uma simples classificação quantitativa; 
A "cultura de avaliação" vem pois dar relevo a aspetos, tais como: a autenticidade, ou seja a aprendizagem situada em contextos reais, ligados à vida profissional; o trabalho de grupo assente na interação social e no feedback, essenciais na comunicação e em processos de pensamento de ordem superior (DIERICK \& DOCHY, 2001). Assume igualmente destaque a autorregulação da aprendizagem por parte do estudante que define e negoceia as tarefas bem como o scaffolding, que apoia o estudante e o faz progredir (McLOUGHLIN \& LUCA, 2001).

Subjacente a esta visão encontra-se uma perspetiva edumétrica que se contrapõe à psicométrica. A abordagem psicométrica centra-se na medida das diferenças entre os estudantes enquanto a perspetiva edumétrica pretende medir o desenvolvimento do próprio aprendente (BRINKE, 2008). Os critérios edumétricos são reconhecidos como válidos e mais justos para a avaliação de competências por enfatizarem a flexibilidade e a autenticidade da avaliação bem como a sua integração no processo de aprendizagem valorizando a sua função formadora (PEREIRA et al. 2010).

A investigação sublinha que os contextos virtuais de aprendizagem podem contribuir para potenciar as capacidades de autorregulação dos estudantes, em particular, a metacognição (VOVIDES et.al. 2007). As práticas nesses contextos concretizam-se em produtos como por exemplo, ensaios, reflexões, apresentações orais (podcast), narrativas digitais (storytelling), artefactos (individuais ou de grupo). Permitem ainda o registo do processo também ele formador e elemento de avaliação (PEREIRA et al., 2009).

\section{O CONCEITO DE AVALIAÇÃO ALTERNATIVA DIGITAL}

Na educação online as estratégias de avaliação são mediadas pela tecnologia. Várias expressões são usadas para referir a avaliação neste cenário, tais como avaliação eletrónica, avaliação online e avaliação digital.

O alargamento na utilização de ferramentas eletrónicas na avaliação, desde os testes de escolha múltipla a questionários, blogs e eportefólios torna ainda mais premente a necessidade de clarificar o entendimento de avaliação eletrónica. Em "Effective assessment in a digital age report" (JISC, 2010), o Joint Information Systems Committee considera que a 
“avaliação eletrónica é, por vezes, entendida somente como avaliação vista no ecrã mas, em sentido lato, pode ser usada para todas as atividades de avaliação tornadas possíveis por meio das tecnologias" (p. 56).

Esta definição surge como muito abrangente. Neste sentido, propõe-se um conceito que incorpora a dimensão tecnológica, mas que é dirigido especificamente para a avaliação de competências. Conforme se esquematiza na figura 1, o conceito de "estratégia de avaliação alternativa digital" (PEREIRA et al., 2010) relativo a um conjunto de propostas de avaliação alternativa, onde o desenho, a execução e o feedback são mediados pelas tecnologias, procura dar resposta a essas exigências. O design implica a definição das competências a avaliar, as indicações sobre a atividade a realizar pelo estudante e o uso de dispositivos eletrónicos para a sua concretização. Em última análise é da responsabilidade do professor, mas poderá incluir contributos dos alunos, tanto na seleção das competências a trabalhar e avaliar, como no tipo de tarefa a desenvolver.

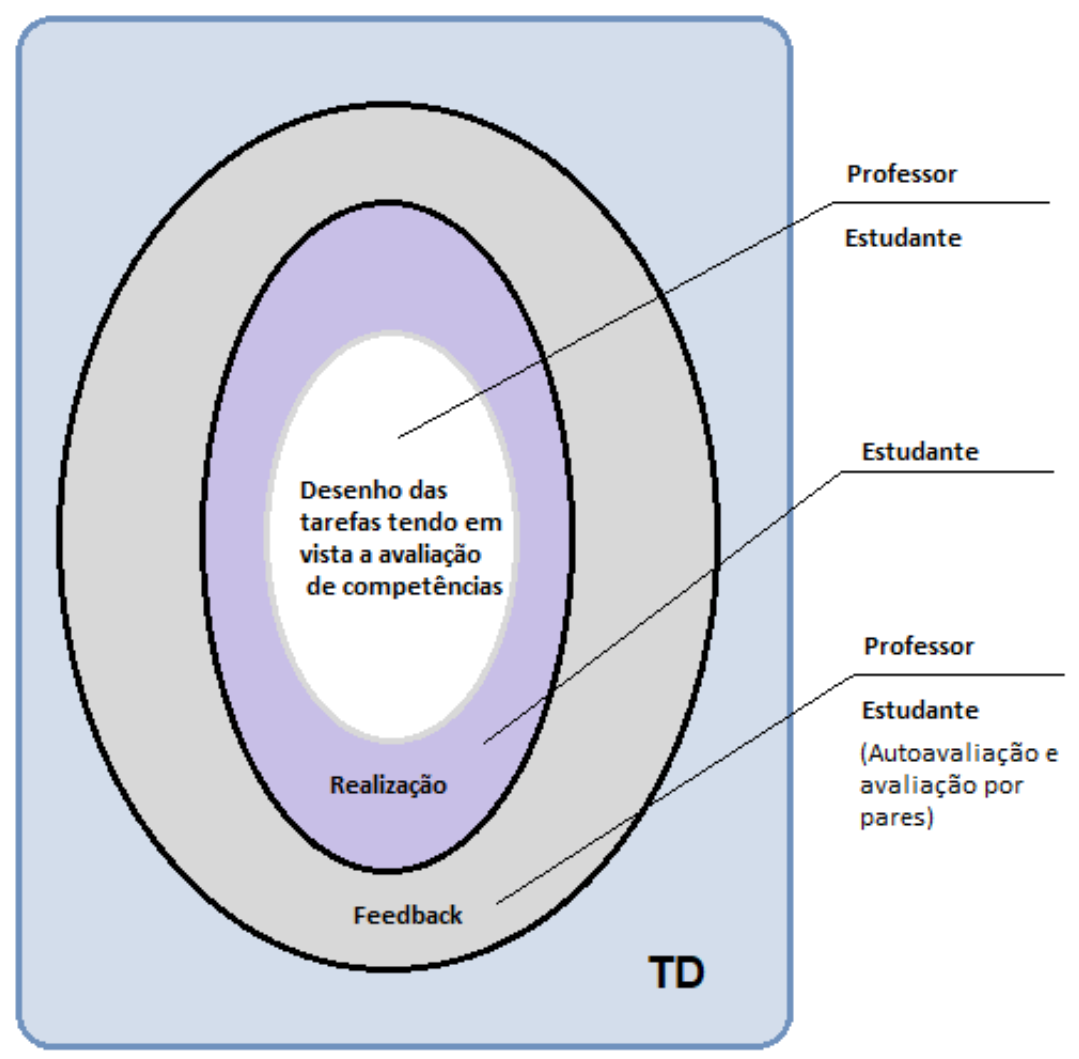

Fig.1- Avaliação alternativa digital: o desenho, a execução e o feedback são mediados pelas tecnologias digitais (TD). 


\section{Revista Docência e Cibercultura}

As diversas realizações do estudante implicam a participação em atividades mediadas pelas tecnologias (como por exemplo e-portefólio, relatório digital, participação em fórum), quer a tarefa seja realizada no computador, na web, ou com o recurso à web, ou recorrendo a dispositivos eletrónicos como tablets, telemóveis, câmaras digitais, etc. A componente de feedback inclui não só os comentários do professor ou tutor, como também a auto e a heteroavaliação por parte dos estudantes.

Uma estratégia de avaliação alternativa digital traduz uma mais-valia no desenvolvimento das competências digitais dos estudantes, particularmente em ambientes de aprendizagem online, caracterizados pela existência de registos sistemáticos. Estes registos funcionam como uma história dos percursos de aprendizagem, em qualquer momento disponíveis e mobilizáveis, quer para a interação dentro do grupo em processos colaborativos na construção de significados e de conhecimento, quer para a reflexão metacognitiva, traduzindo-se num maior envolvimento dos estudantes (PEREIRA, OLIVEIRA \& TINOCA, 2011). Para além disso, sublinha-se a possibilidade de: i) selecionar um momento particular para realização da(s) tarefa(s) de avaliação, ii) permitir uma maior eficiência na disponibilização ao professor e demais participantes, dos produtos resultantes, e iii) incentivar o acesso dos estudantes ao feedback de outros, incluindo do professor, resultando num alargamento de oportunidades que beneficiam o aprofundamento da aprendizagem individual.

\section{MOdelo CONCEPTUAL DE AVALIAÇÃo DIGITAL - Modelo PrACT}

O quadro conceptual proposto por Pereira, Oliveira e Tinoca (2010) teve em conta os desafios colocados pela sociedade do conhecimento no que diz respeito ao desenvolvimento de competências e às instituições de ensino superior, assim como aos novos papeis exigidos a professores e alunos. Este referencial inscreve-se numa perspetiva holística de avaliação e compreende 4 dimensões constituídas cada uma delas por diversos parâmetros que as operacionalizam.

A dimensão praticabilidade que é frequentemente negligenciada, podendo, no entanto, influenciar de modo determinante a escolha da estratégia de avaliação digital a usar, tem a ver com a sua exequibilidade. Implica uma gestão efetiva considerando o tempo

\begin{tabular}{l|l|l} 
v.1 & n.1
\end{tabular}


e o equilíbrio em termos de custo/benefício, quer para os avaliadores quer para as organizações (BROWN, 2004). Importa ainda garantir que os estudantes considerem as tarefas de avaliação realizáveis, relevantes e úteis para a aprendizagem. Para esta dimensão contribuem os seguintes critérios: custos (relacionado com custos de tempo e custos resultantes da utilização de recursos ou investimentos adicionais), eficiência (considera a relação custo-benefício da estratégia de avaliação desenhada para as instituições, professores e estudantes, tendo em conta os resultados esperados) e sustentabilidade (assegura que é possível implementar e sustentar o desenho de avaliação pensado, tendo em conta os perfis dos estudantes e os constrangimentos contextuais, quer das organizações, quer dos avaliadores).

A dimensão autenticidade é reconhecida como fundamental para a avaliação baseada em competências que se pretendem próximas do mundo real/profissional em toda a sua complexidade, reconhecidas como significativas por estudantes, professores e potenciais empregadores. Para esta dimensão concorre um conjunto de critérios que permitem ajuizar do grau de autenticidade da estratégia de avaliação digital, a saber: similitude (relação da estratégia de avaliação digital com o mundo real), complexidade (ligada à natureza das tarefas de avaliação), adequação (respeitante à necessidade de providenciar condições de realização das tarefas de avaliação) e significância (relacionada com o valor significativo da tarefa para professores, estudantes e empregadores).

A dimensão consistência emerge como uma forma de resposta às exigências tradicionais de validade e fiabilidade, requerida pelos indicadores psicométricos. Esta dimensão tem em conta que a avaliação de competências implica a existência de uma variedade de métodos de avaliação, em contextos diversos, de diferentes avaliadores, tal como a adequação das estratégias usadas (DIERICK \& DOCHY, 2001). Salienta a importância de alinhar as competências a avaliar com as tarefas instrucionais e com as estratégias e critérios de avaliação usados, bem como a necessidade de variar os indicadores. Nesta dimensão consideram-se os seguintes critérios: alinhamento instrução-avaliação digital (garante a concordância entre o trabalho desenvolvido durante o percurso de aprendizagem e as tarefas de avaliação), multiplicidade de indicadores (relacionado com o uso de variadas tarefas de avaliação digital e, também, diversos contextos, momentos e avaliadores), 


\section{Revista Docência e Cibercultura}

relevância dos critérios (ligada à pertinência dos critérios usados para a avaliação das competências, individuais ou colaborativas) e alinhamento competências-avaliação digital (relativa à coerência entre as competências a desenvolver e a estratégia de avaliação desenhada).

Por último, a dimensão transparência traduz a necessidade de que a estratégia de avaliação digital seja visível e compreensível por todos os participantes; refere-se assim ao envolvimento dos estudantes, mediante a disponibilização, à partida, de metas e critérios de desempenho e de avaliação, bem como ao reconhecimento dos efeitos positivos que a avaliação deve ter na aprendizagem. Nesta dimensão consideram-se os seguintes critérios: democratização (traduz a disponibilidade e possível participação dos estudantes na definição dos critérios de avaliação), envolvimento (tem a ver com a disponibilidade e possível participação dos estudantes no definir das metas de aprendizagem e das condições de realização das tarefas propostas), visibilidade (remete para a possibilidade de apresentar/partilhar com outros, os seus processos de aprendizagem e/ou os produtos) e impacto (relativo aos efeitos que a estratégia de avaliação digital tem nos processos de aprendizagem e no desenho do programa educacional).

Estas quatro dimensões são, particularmente, relevantes na avaliação de competências em contextos online e ambientes de aprendizagem presenciais fortemente marcados pela utilização das tecnologias digitais.

Na figura 1, representa-se esquematicamente o referencial de avaliação proposto, identificando cada uma das quatro dimensões e os respetivos parâmetros que as consubstanciam. Estas dimensões estão articuladas, assumindo vários graus de interdependência. A praticabilidade, por exemplo, frequentemente negligenciada, pode ter uma influência decisiva no nível de concretização das restantes dimensões.

Este quadro conceptual deu lugar ao modelo PrACT (Praticabilidade, Autenticidade, Consistência e Transparência) podendo ser usado como referencial na definição de uma estratégia alternativa digital para contextos online, híbridos (blendedlearning) ou presenciais com forte utilização das tecnologias. Para além disso, constitui-se como um quadro de referência para a qualidade de uma dada estratégia de avaliação. Os referidos critérios, mais 
do que apenas ilustrar os diferentes traços de cada dimensão, possibilitam uma descrição operacional do grau de realização de cada critério.

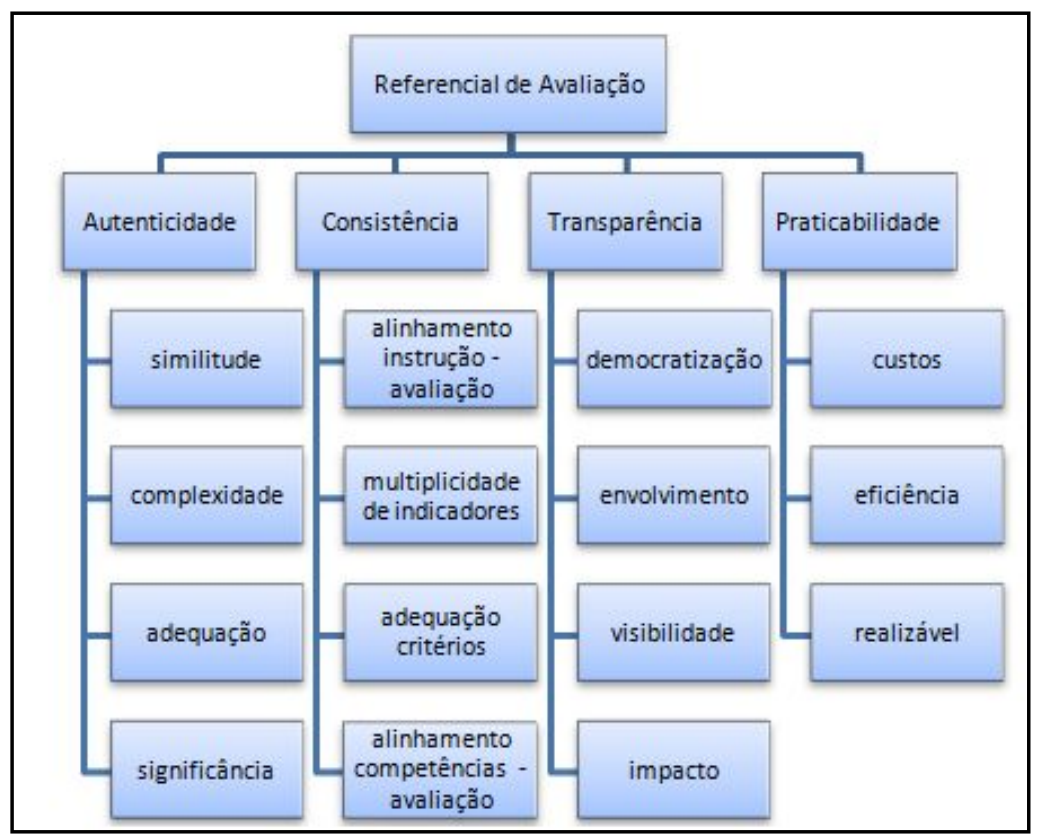

Figura 1 - Dimensões da avaliação alternativa digital - Modelo PrACT

O presente quadro conceptual, que organiza de modo coerente os critérios a aplicar no conjunto das quatro dimensões referidas, tem vindo a ser aplicado em unidades curriculares de diversos cursos em oferta na Universidade Aberta (OLIVEIRA \& AMANTE, 2014), bem como no desenvolvimento de oficinas de formação em outras instituições (AMANTE, OLIVEIRA \& ARAÚJO, 2017) contribuindo para a sua validação e consequente aperfeiçoamento. Pretende-se assim dar um contributo relevante que colabore na resposta aos desafios que se colocam à aprendizagem e à avaliação na sociedade atual.

\section{CONLUSÃO}

Apresentámos o quadro conceptual subjacente ao modelo PrACT para avaliação digital, desenvolvido com base numa perspetiva edumétrica, que se contrapõe às abordagens psicométricas tradicionais para a avaliação. Argumenta-se que a avaliação sustentável (BOUD, 2000) para ambientes de aprendizagem tecnicamente enriquecidos 


\section{Revista Docência e Cibercultura}

apoia o desenvolvimento das competências de autorregulação dos estudantes ajudando-os a planear, monitorar e controlar melhor a sua própria aprendizagem. Estas competências de planeamento e de monitorização são fundamentais num contexto de aprendizagem ao longo da vida na sociedade digital, onde a maioria dos indivíduos passará por várias profissões ao longo da sua vida profissional.

O quadro conceptual apresentado confirma um novo entendimento do ensino e da aprendizagem, tendo na sua origem a reflexão em torno da educação online e a experiência das autoras como docentes e investigadoras em contextos virtuais de aprendizagem.

O PrACT contribui para a definição de um padrão de qualidade das estratégias de avaliação em diversos níveis de ensino e contextos, sejam eles totalmente virtuais, híbridos ou mesmo presenciais. A sua aplicação permitir ao docente sustentar o desenho instrucional das suas disciplinas, assente num conjunto de princípios que promovem a integração da avaliação na aprendizagem (assessment for learning) e a relação desta com a vida real e profissional, bem como o envolvimento dos estudantes no processo, deixando assim de ser algo exterior à sua aprendizagem. Simultaneamente, com base neste quadro conceptual, a instituição dispõe de um padrão que lhe permite analisar e avaliar a qualidade das práticas pedagógicas dos seus cursos, considerando as dimensões enunciadas e os respetivos critérios.

\section{REFERÊNCIAS}

AMANTE, L. A Avaliação das Aprendizagens em Contexto Online: O e-portefólio como Instrumento Alternativo. In Paulo Dias \& António Osório (Orgs.) Aprendizagem (In)Formal na Web Social. Centro de competência da Universidade do Minho, Braga, 2011.

AMANTE, OLIVEIRA \& ARAÚJO. O Modelo PrACT: Um novo olhar sobre a conceção e desenvolvimento de práticas de avaliação alternativa digital. Oficina realizada no âmbito do V Seminário Web Currículo, PUC São Paulo, 16 a 18 de Outubro de 2017.

BAARTMAN, L.K.J., BASTIENS, T.J., KIRSCHNER, P.A. \& VLEUTEN. Evaluating assessment quality in competence-based education: A qualitative comparison of two frameworks. Educational Research Review, 2, 114-129, 2007. 
BIRENBAUM, M. Assessment 2000: Towards a pluralistic approach to assessment. In M. Birenbaum \& F. J. R. C. Dochy (Eds.), Alternatives in assessment of achievement, learning processes and prior knowledge, Boston: Kluwer Academic Publishers, 3-29, 1996.

BOUD, D. Enhancing Learning through Self Assessment. London: Kogan Page, 1995).

BOUD, D. Sustainable assessment: rethinking assessment for the learning society, Studies in Continuing Education, 22(2), 151-167, 2000.

BROWN, S. Assessment for learning. Learning and Teaching in Higher Education. 1, 81-89, 2004.

BRINKE, D. Assessment of prior learning. Maastricht, the Netherlands: Datawyse, 2008.

DIERICK, S., \& DOCHY, F. J. R. C. New lines in edumetrics: new forms of assessment lead to new assessment criteria. Studies in Educational Evaluation, 27, 307-329, 2001.

DOCHY, F. A new assessment era: Different needs, new challenges. Learning and instruction, 10 (suppl. 1), 11-20, 2001.

FALCHIKOV, N. Improving assessment through student involvement . London: Routledge Falmer), 2005.

FERNANDES, D. Avaliação das Aprendizagens. Uma Agenda, Muitos Desafios. Lisboa: Texto Editora, 2004.

FIGUEIREDO, A. D. Por uma escola com futuro ... para além do digital. Revista Nova Ágora, no 5, Set. 2016, p. 19-21. 2016.

GOMES, M. J. Problemáticas da avaliação na educação online. In M. Silva, L Pesce e A. Zuin (Orgs.) Educação online: cenário, formação e questões didáctico-metodológicas (pp. 309-335). São Paulo: Wak editor, 2010.

GULIKERS, J. T. M., BASTAENS, T. J., \& KIRSNHER, P. A. A five-dimensional framework for authentic assessment. Educational Technology Research and Design, 53, 67-87, 2004.

HADJI, C. L'évaluation démystifiée. Paris: ESF éditeur, 1997.

HERRINGTON, J. \& HERRINGTON, A. Authentic Assessment and Multimedia: how university students respond to a model of authentic assessment. Higher Education Research \& Development, 17(3), 305 - 322, 1998. 


\section{Revista Docência e Cibercultura}

JOINT INFORMATION SYSTEMS COMMITTEE. Effective assessment in a digital age report, 2010.

McCONNELL, D. E-learning Groups and Communities. Berkshire: Open University Press, 2006.

MacLELLAN, E. How convincing is alternative assessment for use in higher education? Assessment \& Evaluation in Higher Education. 29(3), 311 - 321, 2004.

McDOWELL, L. The impact of innovative assessment on student learning. Education and Training International, 32, 302-313, 1995.

McLOUGHLIN, C. \& LUCA, J. Quality in online delivery: what does it mean for assessment in e-learning environments?. In Meeting at the crossroads. Proceedings of the 18th Annual Conference of the Australasian Society for Computers in Learning in Tertiary Education. Melbourne, Australia, 9-12 December 2001.

OLIVEIRA, I.; AMANTE, L. "Alternative assessment in online learning environments in Higher Education" In I/ Congresso Internacional de Ciencias de la Educación y del Desarrollo, Granada, 2014.

PEREIRA, A; QUINTAS-MENDES, A; MORGADO, L.; AMANTE, L.; BIDARRA, J. Modelo Pedagógico Virtual da Universidade Aberta, Universidade Aberta, Lisboa, 2007.

PEREIRA, A.; OLIVEIRA, I. TINOCA, L.; AMANTE, L.; RELVAS, M. J.; PINTO, M. C.; MOREIRA, D. "Evaluating continuous assessment quality in competence-based education online: the case of the e-folio ". In Proceedings of EDEN - European Distance and Elearning Network Annual Conference, Gdansk, Poland, 2009 (em colaboração) EDEN 2009 Best Research Paper Award disponível em http://www.edenonline.org/eden.php?menuld=7\&contentld=835 Acesso eem 7 de julho de 2017.

PEREIRA, A., OLIVEIRA, I. \& TINOCA. A Cultura de Avaliação: que dimensões? In Fernando Costa, Guilhermina Miranda, João Matos Isabel Chagas \& Elisabete Cruz (Eds.). Actas do I Encontro Internacional TIC e Educação: TICeduca 2010. Lisboa, Novembro 2010.

PEREIRA, A., OLIVEIRA, I. \& TINOCA, L. Assessment Culture dimensions: contributions for quality development. EARLI 2011 conference in Exeter, UK, 2011. 
PEREIRA, A.; OLIVEIRA; I; AMANTE, L. Fundamentos da avaliação alternativa digital. In T. Cardoso, A. Pereira e L.Nunes (Eds.) Avaliação e Tecnologias no Ensino Superior. Ebook, LE@D, Universidade Aberta, Lisboa, 2015.

PINTO, J. \& SANTOS, L. Modelos de avaliação das aprendizagens. Lisboa: Universidade Aberta, 2006.

PINTO, J. A avaliação em educação: Da linearidade dos usos à complexidade das práticas", In L.Amante \& I.Oliveira (coords) Avaliação das Aprendizagens: Perspetivas, contextos e práticas. Lisboa: Le@D, Universidade Aberta (3-40), 2016. Disponível em: https://goo.gl/IWwZc8 Acesso em 28 de Setembro de 2017.

SLUIJSMANS, D. M. A., PRINS, F., MARTENS, R. A framework for integrated performance assessment in E-Learning. Learning Environments Research, 9(1), 45-66, 2006.

TINOCA, L., OLIVEIRA, I. \& PEREIRA, A. Group work peer-assessment in an online environment.In European Association of Distance Teaching Universities Conference, 2007. Disponível em: http://www.eadtu.nl/conference-2007/files/SAA4.pdf. Acesso em 7 de Setembro de 2017.

VOVIDES, Y., SANCHEZ-ALONSO, S., MITROPOULOU, V. and NICKMANS, G. The use of elearning course management systems to support learning strategies and to improve self-regulated learning. Educational Research Review, 2, 64 - 74, 2007. 